\title{
Catálogo de las Especies de Sphingidae (Lepidoptera) en Honduras
}

\author{
Ana Clariza Samayoa ${ }^{1}$ y Ronald D. Cave $^{2}$
}

Resumen: Se presenta un catálogo de las 107 especies de la familia Sphingidae en Honduras. Treinta y tres especies son registros nuevos para el país. Se reportan datos de distribución geográfica, estacionalidad de los adultos, y plantas hospederas de las larvas. Se analiza la diversidad de la familia en todos los departamentos nacionales, en cuatro parques nacionales, y por gradientes altitudinales. Fotografías de 76 especies son presentadas.

Palabras clave: Gusano cachudo, nuevos registros de país, estacionalidad, parques nacionales.

Abstract: A catalogue of the 107 species of Sphingidae known to occur in Honduras is presented. Thirty-three species are new country records. Geographic distribution, adult seasonality, and larval host plant data are reported. The diversity of the family in all national departments, in four national parks, and by altitudinal gradients is analyzed. Photographs of 76 species are presented.

Key words: Hornworm, new country records, seasonality, national parks.

\section{Introducción}

Las especies de la familia Sphingidae son poderosos voladores cuyos adultos se caracterizan por poseer las alas anteriores alargadas y usualmente un cuerpo robusto. Poseen una larga proboscis que utilizan para alimentarse del néctar de ciertas flores, por lo que se les puede confundir con colibríes. Algunas especies de hábitos diurnos se diferencian por sus alas transparentes. Los inmaduros se reconocen fácilmente por la presencia de un cuerno en el octavo segmento abdominal (Figura 1). Las larvas de ciertas especies adoptan una posición de defensa inusual ya que elevan las patas manteniendo las propatas en la superficie en que se encuentren.

La familia Sphingidae está dividida en tres subfamilias: Sphinginae, Smerinthinae y Macroglossinae. Se conocen aproximadamente 1,100 especies alrededor del mundo (Arnett 1985). Para Honduras, Lehman (1971) listó 15 géneros y 34 especies colectados en las cercanías de cinco ciudades: La Ceiba, San Pedro Sula, La Lima, El Zamorano y Tegucigalpa (Figura 2). Oehlke (2007) cita 71 especies. En el presente catálogo se listan las especies conocidas en Honduras y se reportan datos de distribución geográfica, estacionalidad de los adultos, y plantas hospederas para las larvas de ciertas especies. Se analiza la diversidad de la familia en todos los departamentos nacionales, en cuatro parques nacionales, y por gradientes altitudinales. Se presentan también fotografías de 76 ejemplares depositados en la Colección de Artrópodos de la Escuela Agrícola Panamericana, Zamorano, Honduras.

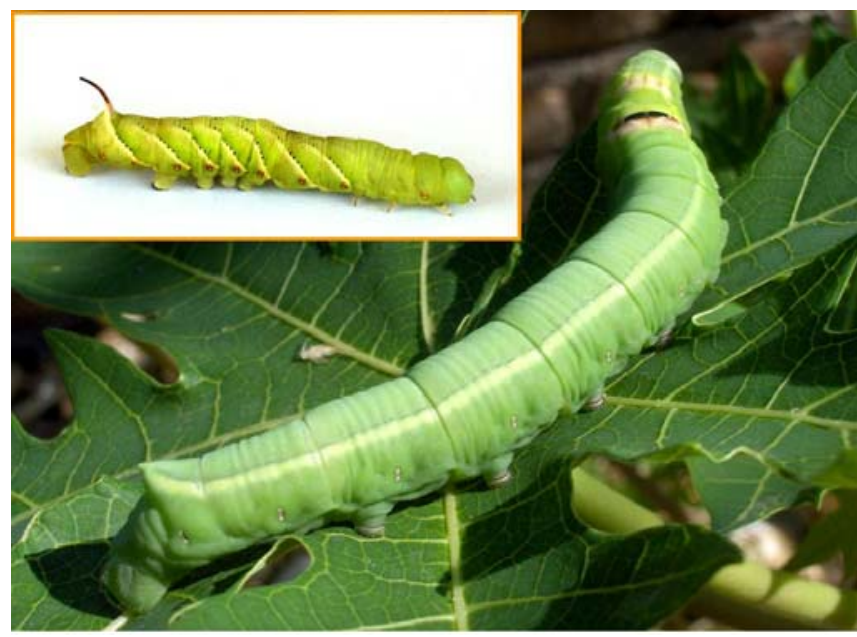

Figura 1. Larva de Sphingidae alimentándose de hoja de papaya (Carica papaya). Foto inserta, Manduca sexta con el cuerno en el octavo segmento abdominal.

\footnotetext{
${ }^{1}$ Colonia Tiloarque, casa 1716 primera etapa, calle principal, Tegucigalpa, Honduras, anaclariza@gmail.com

${ }^{2}$ Indian River Research and Education Center, University of Florida, 2199 South Rock Road, Fort Pierce, FL 34945, rdcave@ufl.edu
} 


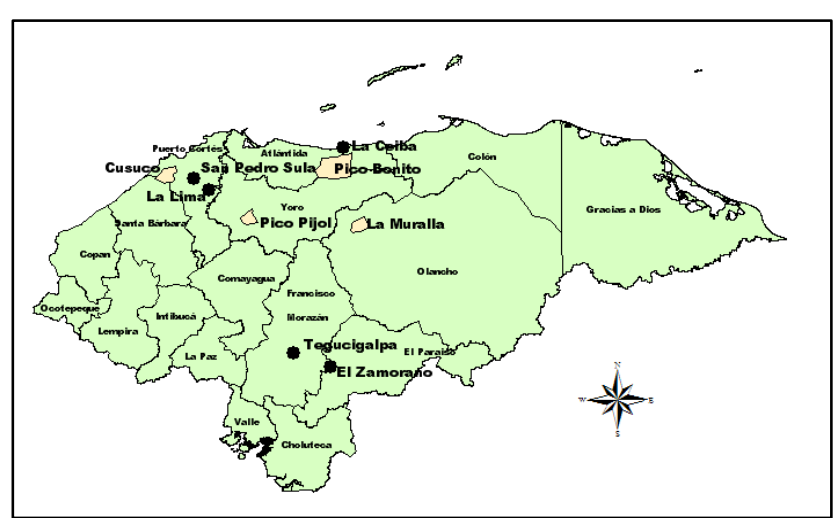

Figura 2. Mapa de Honduras y ubicación de los cuatro parques nacionales con mayor número de colectas.

\section{Materiales y Métodos}

Los adultos se colectaron con ayuda de lámparas de vapor de mercurio. Se les inyectó alcohol al 70\% con una jeringa de punta fina, se depositaron en sobres y se almacenaron en una caja plástica para protección. Las lámparas se mantuvieron encendidas desde las seis de la tarde hasta el amanecer, en un campo abierto rodeado por o frente al bosque. Las lámparas se vigilaron durante toda la noche.

Las colectas se realizaron principalmente en áreas protegidas de Honduras, con énfasis en: Parque Nacional La Muralla, Parque Nacional Pico Bonito, Parque Nacional Pico Pijol y Parque Nacional Cusuco (Figura 2). El Parque Nacional La Muralla (1,4002,064 msnm) tiene una extensión de 21,035 ha con bosque tropical siempreverde latifoliado montano y cafetales. El Parque Nacional Pico Bonito (150-2,436 msnm) posee una área de unas 112,500 ha con un mosaico de bosque latifoliado submontano, bosque tropical siempreverde mixto (bosque latifoliado con pinos) montano, bosque tropical siempreverde latifoliado montano, y bosque tropical siempreverde latifoliado de tierras bajas bien drenadas. El Parque Nacional Pico Pijol (1,300-2,282 msnm) posee bosque tropical siempreverde latifoliado montano en 18,000 ha rodeadas por cafetales. El Parque Nacional Cusuco (1,400-2,242 msnm) tiene 9,000 ha cubiertas por bosque tropical siempreverde mixto montano con cafetales adyacentes.

Las identificaciones de los especimenes en las colecciones del Museo de Mariposas en La Ceiba y de la Escuela Agrícola Panamericana se realizaron con el libro “Sphingidae Mundi” (D' Abrera 1986) y referencias visuales de Maes y Schmit (1999), Janzen y Hallwachs (2004), y Oehlke (2007) en la Internet. La nomenclatura de especies sigue aquella de Kitching y Cadiou (2000).

Las especies del catálogo se organizan por subfamilia y tribu. Dentro de cada tribu se listan los géneros en orden alfabético; dentro de cada género se listan las especies en orden alfabético. La asignación de los géneros a sus tribus se hizo según los sitios Web mencionados arriba. Por cada especie, se listan los departamentos de Honduras (Figura 2) en los que se le ha recolectado; nuevos registros para el país son indicados. Los meses de captura de adultos son listados y se proveen las elevaciones de capturas (mínimo-máximo). La distribución mundial de la especie es indicada con la cita bibliográfica; en algunos casos donde esta información es dudosa, está precedida por el signo de interrogación “?”. Información ecológica, como enemigos naturales y plantas hospederas de la larva, está incluida cuando se conoce esta información. Las notas de interés se acompañan con su cita bibliográfica, aunque no todas las especies poseen esta información completa.

\section{Subfamilia Macroglossinae Harris, 1839 Tribu Dilophonotini Burmeister, 1878}

\section{Aellopos Hübner, 1819}

ceculus (Cramer, 1777) (Lámina I)

Departamentos: Atlántida (Nuevo registro para Honduras)

Meses de captura: Marzo

Altura: $850 \mathrm{msnm}$

Distribución: México al sur de Brasil, Las Antillas (D’ Abrera 1986)

clavipes Rothschild \& Jordan, 1903

Distribución: sur de Estados Unidos a Argentina (Monzón Sierra y Haxaire 2006)

Nota: especie citada por Oehlke (2007)

titan (Cramer, 1777) (Lámina I)

Departamentos: Francisco Morazán, Cortes

Meses de captura: Mayo, Julio

Altura: 185-1600 msnm

Distribución: sur de los Estados Unidos hasta el norte de Argentina, Uruguay, Las Antillas (D’Abrera, 
1986)

Hospederos de la larva: especies de la familia Rubiaceae (D’ Abrera 1986)

\section{Aleuron Boisduval, 1870}

chloroptera (Perty, 1833) (Lámina I)

Departamentos: Atlántida (Nuevo registro para

\section{Honduras)}

Meses de captura: Mayo

Altura: $185 \mathrm{msnm}$

Distribución: México al sur de Brasil (Monzón Sierra

y Haxaire 2006)

neglectum Rothschild \& Jordan, 1903 (Lámina I)

Departamentos: Atlántida (Nuevo registro para

Honduras)

Meses de captura: Marzo

Altura: 35-185 msnm

Distribución: México al sur de Brasil, Bolivia (D' Abrera 1986)

\section{Callionima Lucas, 1857}

denticulata (Shaus, 1895) (Lámina I)

Departamentos: Atlántida, Cortés

Meses de captura: Julio

Altura: 36-1600 msnm

Distribución: México a Bolivia (D’ Abrera 1986)

Notas: Se le caracteriza por la forma dentada del ala anterior (D'Abrera 1986)

falcifera (Gehlen, 1943) (Lámina I)

Distribución: Atlántida, Colón, Francisco Morazán, Yoro, Olancho

Meses de captura: Mayo, Septiembre

Altura: 175-1215 msnm

Distribución: Costa Rica a Venezuela, Guyana (D’

Abrera 1986)

Hospederos de la larva: Stemmadenia (Apocynaceae) (Maes 1998)

nomius Walker, 1856

Departamentos: Atlántida (Nuevo registro para Honduras)

Meses de captura: Mayo, Agosto

Altura: 550-1215 msnm

Distribución: México al sur de Brasil y Paraguay

(Monzón Sierra y Haxaire 2006)

pan (Cramer, 1779)

Departamentos: Atlántida

Meses de captura: Marzo, Abril, Mayo

Distribución: Brasil (D’Abrera 1986)

parce (F., 1775) (Lámina I)
Departamentos: Atlántida, Cortés, Yoro

Meses de captura: Marzo, Julio, Septiembre

Altura: 60-1160 msnm

Distribución: Florida (Estados Unidos) al sur de Brasil, Bolivia (D’ Abrera 1986)

Hospederos de la larva: Rosa (Rosaceae), Vitis, Cissus, Ampelopsis (Vitaceae) (Maes 1998)

Enyo Hübner, 1819

gorgon (Cramer, 1777) (Lámina I)

Departamentos: Atlántida, Cortés, Francisco Morazán, Gracias a Dios, Lempira (Nuevo registro para Honduras)

Meses de captura: Marzo, Abril, Mayo, Agosto

Altura: 5-2050 msnm

Distribución: México al sur de Brasil, Paraguay (D' Abrera 1986)

lugubris lugubris (L., 1771) (Lámina I)

Departamentos: Atlántida, Cortés, Francisco

Morazán, Yoro

Meses de captura: Abril, Mayo, Julio, Septiembre

Altura: 850-1600 msnm

Distribución: sur de los Estados Unidos hasta el norte de Argentina, Uruguay, Las Antillas (a excepción de Jamaica) (D’ Abrera 1986)

Hospederos de la larva: Vitis, Cissus, Ampelopsis (Vitaceae) (Maes 1998)

Notas: El color de la larva puede ser verde azulado pálido o blanco verdoso, con un par de bandas dorsolaterales, bandas blancas oblicuas recorriendo desde la cabeza a la base del cuerno. La larva se transforma en pupa dentro de celdas bajo la superficie del suelo (Moss 1920)

ocypete (L., 1758) (Lámina I)

Departamentos: Atlántida, Cortés, Francisco Morazán, Gracias a Dios, Olancho, Santa Bárbara, Yoro

Meses de captura: Marzo, Abril, Mayo, Julio, Agosto, Septiembre

Altura: 1-1600 msnm

Distribución: Florida (Estados Unidos) a Paraguay, Las Antillas (D’ Abrera 1986)

Hospederos de la larva: Tetracera (Dilleniaceae), Cissus (Vitaceae) (Maes 1998)

taedium Schaus, 1890

Distribución: sur de México al sur de Brasil (Monzón Sierra y Haxaire 2006)

Nota: especie citada por Oehlke (2007) 
Erinnyis Hübner, 1819

alope (Drury, 1773) (Lámina I)

Departamentos: Atlántida

Meses de captura: Julio

Altura: $20 \mathrm{msnm}$

Distribución: sur de los Estados Unidos hasta el

norte de Argentina, Uruguay, Las Antillas (D'

Abrera 1986)

Hospederos de la larva: Allamanda (Apocynaceae),

Carica (Caricaceae), Manihot, Jatropha (Euphorbiaceae), Ficus (Moraceae) (Maes 1998).

Nota: Enemigos naturales son Cotesia congregata

(Say) (Braconidae), Derostenus sp. (Eulophidae),

Eupelmus sp. (Eupelmidae) y Telenomus sp. (Scelionidae) (Maes 1998). El último estadío larval es de color marrón amarillento oscuro con bandas irregulares amarillas debajo de las propatas (Moss 1912).

crameri (Schaus, 1898) (Lámina I)

Departamentos: Atlántida, Comayagua, Francisco

Morazán

Meses de captura: Febrero, Abril, Mayo

Altura: $850 \mathrm{msnm}$

Distribución: sur de Estados Unidos a Paraguay, Las

Antillas (D’ Abrera 1986)

Hospederos de la larva: Allamanda,

Tabernaemontana (Apocynaceae), Carica

(Caricaceae) (Maes 1998)

ello ello (L., 1758) (Lámina II)

Departamentos: Atlántida, Cortés, Francisco

Morazán, Olancho, Yoro

Meses de captura: Enero, Mayo, Junio, Julio, Agosto,

Septiembre

Altura: 2-1600 msnm

Distribución: Canadá a Argentina, Uruguay, Las

Antillas, Chile? (D’ Abrera 1986)

Hospederos de la larva: Manihot, Sebastiana, Sapium, Euphorbia, Poinsettia, Cnidoscolus (Euphorbiaceae), Yucca (Agavaceae), Manilkara (Sapotaceae) (Maes 1998)

Nota: Cotesia congregata es un enemigo natural de la larva (Cave 1995).

lassauxii (Boisduval, 1859)

Departamentos: Atlántida, Olancho

Meses de captura: Febrero, Abril, Julio

Altura: 10-300 msnm

Distribución: sur de los Estados Unidos al sur de

Brasil, Bolivia, norte de Argentina, Paraguay?, Las Antillas (D’ Abrera 1986)
Hospederos de la larva: Carica papaya L.

(Caricaceae) y varias especies de la familia

Asclepiadaceae (D’ Abrera 1986)

Nota: Durantes las últimas etapas larvales es verde amarillento oscuro, con un par de líneas dorsolaterales del tercer al onceavo segmento (Moss 1920).

obscura Fabricius, 1775

Distribución: Estados Unidos a Argentina (Monzón

Sierra y Haxaire 2006)

Nota: especie citada por Oehlke (2007)

oenotrus (Cramer, 1780) (Lámina II)

Departamentos: Atlántida, Colón, Cortés, Gracias a

Dios, Yoro, Olancho

Meses de captura: Enero, Abril, Junio, Julio,

Septiembre

Altura: 2-1600 msnm

Distribución: sur de los Estados Unidos al norte de

Argentina, Uruguay, Las Antillas (D’ Abrera 1986)

Hospederos de la larva: especies de la familia

Apocynaceae (Moss 1920)

yucatana (Druce, 1888)

Distribución: México a Costa Rica (Monzón Sierra y

Haxaire 2006)

Nota: especie citada por Oehlke (2007)

Hemeroplanes Hübner, 1819

ornatus Rothschild, 1894 (Lámina II)

Departamentos: Atlántida (Nuevo registro para

Honduras)

Meses de captura: Marzo

Altura: $20 \mathrm{msnm}$

Distribución: Colombia al centro de Brasil (D'

Abrera 1986)

Nota: La larva se asemeja a la serpiente del género Bothrops (D’ Abrera 1986).

triptolemus (Cramer, 1779) (Lámina II)

Departamentos: Atlántida, Cortés, Francisco

Morazán, Olancho (Nuevo registro para

Honduras)

Meses de captura: Enero, Abril, Mayo, Junio

Altura: 5-850 msnm

Distribución: México a Brasil (D’ Abrera 1986)

Isognathus G. Felder \& R. Felder, 1862

rimosa (Grote, 1865)

Distribución: México, Brasil, Centro América. Cuba

(Oehlke, 2007)

Nota: especie citada por Oehlke (2007) 
Nyceryx Boisduval, 1875

coffeae (Walker, 1856) (Lámina II)

Departamentos: Atlántida, Cortés, Olancho (Nuevo registro para Honduras)

Meses de captura: Junio

Altura: 420-700 msnm

Distribución: Belice a Brasil (D’ Abrera 1986)

eximia (Rothschild \& Jordan, 1916) (Lámina II)

Departamentos: Cortés, Yoro (Nuevo registro para

Honduras)

Meses de captura: Mayo

Altura: 1450-1500 msnm

Distribución: Panamá (D’’ Abrera 1986)

riscus (Schaus, 1890)

Departamentos: Atlántida

Meses de captura: Abril

Altura: $1450 \mathrm{msnm}$

Distribución: México a Bolivia (Monzón Sierra y

Haxaire 2006)

tacita (Druce, 1888) (Lámina II)

Departamentos: Atlántida (Nuevo registro para

Honduras)

Meses de captura: Enero

Altura: 35-185 msnm

Distribución: México a Bolivia (D’ Abrera 1986)

Notas: La franja blanca del tergito abdominal y los puntos gris plateados en las alas son características para distinguir esta especie (D’ Abrera 1986).

Madoryx Boisduval, 1874

oiclus (Cramer, 1779) (Lámina II)

Departamentos: Francisco Morazán

Meses de captura: Mayo

Altura: $850 \mathrm{msnm}$

Distribución: México al sureste de Brasil, Trinidad

(D’ Abrera 1986)

plutonius (Hübner, 1819) (Lámina II)

Departamentos: Atlántida, Cortés, Santa Bárbara, Yoro (Nuevo registro para Honduras)

Meses de captura: Enero, Abril, Mayo, Septiembre

Altura: 2-1215 msnm

Distribución: México a Bolivia y el sur de Brasil (D’

Abrera 1986)

Pachylia Walker, 1856

darceta Druce ,1881 (Lámina II)

Departamentos: Atlántida, Gracias a Dios, Yoro (Nuevo registro para Honduras)

Meses de captura: Enero, Mayo, Septiembre
Altura: 1-640 msnm

Distribución: Panamá a las Amazonas, Bolivia (D' Abrera 1986)

ficus (L.1758) (Lámina III)

Departamentos: Atlántida, Cortés, Francisco

Morazán, Yoro

Meses de captura: Enero, Marzo, Mayo, Agosto, Julio, Septiembre, Noviembre, Diciembre

Altura: 2-1000 msnm

Distribución: sur de Estados Unidos al sur de Brasil, Uruguay (D' Abrera 1986)

Hospederos de la larva: Ficus, Cecropia, Brosimum, Chlorophora, Castilla (Moraceae) (Maes 1998)

syces syces (Hübner, 1819) (Lámina III)

Departamentos: Atlántida, Cortés, Francisco

Morazán, Yoro

Meses de captura: Mayo, Noviembre

Altura: 185-850 msnm

Distribución: México al sur de Brasil, Uruguay (D’

Abrera 1986)

Hospederos de la larva: Moraceae (Maes 1998)

Pachylioides Hodges, 1971

resumens (Walker, 1856) (Lámina III)

Departamentos: Atlántida, Cortés, Francisco Morazán, Gracias a Dios, Olancho, Santa Bárbara, Yoro

Meses de captura: Marzo, Mayo, Junio, Septiembre

Altura: 5-1450 msnm

Distribución: sur de los Estados Unidos a Uruguay,

Paraguay, norte de Argentina, Las Antillas. (D'

Abrera 1986)

Hospederos de la larva: Echites (Apocynaceae) (Maes 1998)

Perigonia Herrich-Schäffe, 1854

jamaicensis Rothschild, 1894

Departamentos: Atlántida, Colón, Cortés (Nuevo registro para Honduras)

Meses de captura: Abril, Junio

Altura: 10-1500 msnm

Distribución: Jamaica

lusca (F. 1777) (Lámina III)

Departamentos: Atlántida, Colón, Cortés, Francisco

Morazán, Olancho, Yoro

Meses de captura: Marzo, Abril, Mayo, Junio,

Septiembre

Altura: $150 \mathrm{msnm}$

Distribución: sur de los Estados Unidos a Argentina, 
Uruguay, Las Antillas, (D’ Abrera 1986)

Hospederos de la larva: Calycophyllum, Coffea, Guettarda (Rubiaceae) (Maes 1998)

stulta Herrich-Schäffer, 1854 (Lámina III)

Departamentos: Cortés, Olancho, Yoro (Nuevo registro para Honduras)

Meses de captura: Junio, Septiembre

Altura: 1450-1600 msnm

Distribución: Guatemala a Brasil (D’ Abrera 1986)

Phryxus Hübner, 1819

caicus (Cramer, 1777) (Lámina III)

Departamentos: Atlántida

Meses de captura: Septiembre

Altura: 185 msnm

Distribución: sur de los Estados Unidos hasta el sur de Brasil y norte de Argentina (D’ Abrera 1986)

Pseudosphinx Burmeister, 1855

tetrio (L., 1771) (Lámina III)

Departamentos: Atlántida, Francisco Morazán

Meses de captura: Abril, Mayo

Altura: 35-850 msnm

Distribución: sur de los Estados Unidos a Paraguay,

Uruguay, Las Antillas.

Hospederos de la larva: Plumeria, Jasminum (Euphorbiaceae) (Carter 1992), Nerium oleander L., Tabernaemontana australis Müll. Arg. (Apocynaceae) (D’ Abrera 1986), Ficus (Moraceae) (Maes 1998)

Nota: La larva está marcada con bandas negras y amarillo brillante; la cabeza es marrón oscuro (Moss 1912).

Stolidoptera Rothschild \& Jordan, 1903

tachasara (Druce, 1888) (Lámina III)

Altura: 35-1160 msnm

Departamentos: Atlántida, Comayagua, Cortés, Francisco Morazán, Olancho, Yoro (Nuevo registro para Honduras)

Meses de captura: Enero, Abril, Mayo, Junio, Julio, Septiembre

Distribución: México a Venezuela (D’ Abrera 1986)

Unzela Walker, 1856

japix japix (Cramer, 1776) (Lámina III)

Altura: $185 \mathrm{msnm}$

Departamentos: Atlántida (Nuevo registro para Honduras)
Meses de captura: Julio

\section{Tribu Macroglossini Harris, 1839}

Cautethia Grote, 1865

spuria (Boisduval, 1875)

Departamentos: Cortés

Meses de captura: Junio

Altura: $1500 \mathrm{msnm}$

Distribución: México

Hospederos de la larva: Exostemma, Coutarea hexandra (Jacq.) K. Schum. (Rubiaceae) (Maes y Schmit 1999)

yucatana Clark, 1919

Distribución: centro de México a Panamá (Monzón

Sierra y Haxaire 2006)

Nota: especie citada por Oehlke (2007)

Hyles Hübner, 1819

lineata lineata (F., 1775) (Lámina IV)

Altura: 850-1450 msnm

Departamentos: Francisco Morazán, Ocotepeque, Olancho.

Meses de captura: Mayo, Agosto

Distribución: Norte y Sur América, Europa, África, Asia y Australia. (Carter 1992)

Hospederos de la larva: Manihot (Euphorbiaceae), Phaseolus (Fabaceae), Asphodelus (Liliaceae), Gossypium (Malvaceae), Mirabilis, Boerhaavia (Nyctaginaceae), Epilobium, Fucsia (Onagraceae), Sesamum (Pedaliaceae), Rumex, Polygonum, (Polygonaceae), Portulaca, (Portulacaceae), Tríbulus, Zygophyllum (Zygophyllaceae) (Maes 1998)

Nota: El parasitoide Brachymeria flavipes (F.) (= robusta Cresson) (Chalcididae) se reporta como enemigo natural (Maes 1998)

Xylophanes Hübner, 1819

acrus Rothschild \& Jordan, 1910 (Lámina IV)

Altura: $175 \mathrm{msnm}$

Departamentos: Atlántida (Nuevo registro para Honduras)

Meses de captura: Mayo

amadis (Stoll, 1782)

Departamentos: Atlántida, Olancho (Nuevo registro

para Honduras)

Meses de captura: Abril, Julio, Octubre

Altura: $1420 \mathrm{msnm}$ 
Distribución: México, Nicaragua, Panamá, Colombia (Maes 1998)

anubus (Cramer, 1777) (Lámina IV)

Departamentos: Atlántida, Yoro

Meses de captura: Marzo, Mayo, Septiembre

Altura: 35-1450 msnm

Distribución: México a Paraguay (Monzón Sierra y

Haxaire, 2006)

Hospederos de la larva: Psychotria (Rubiaceae) (Maes 1998)

belti (Druce, 1878) (Lámina IV)

Departamentos: Atlántida, Cortés, Gracias a Dios, Yoro (Nuevo registro para Honduras)

Meses de captura: Febrero, Mayo, Agosto, Septiembre

Altura: 35-1600 msnm

Distribución: México a Panamá (Monzón Sierra y

Haxaire 2006)

ceratomioides Grote \& Robinson, 1867 (Lámina IV)

Altura: 35-2000 msnm

Departamentos: Atlántida, Comayagua, Copán, Cortés, Olancho, Santa Bárbara, Yoro

Meses de captura: Enero, Marzo, Abril, Junio, Julio, Agosto, Septiembre

Distribución: México, Nicaragua, Venezuela, Guyana Francesa, Brasil, Argentina (Maes 1998)

chiron nechus (Cramer, 1777) (Lámina IV)

Departamentos: Atlántida, Cortés, Francisco Morazán, Lempira, Ocotepeque, Olancho, Yoro

Meses de captura: Enero, Mayo, Junio, Agosto, Septiembre

Altura: 35-1600 msnm

Hospederos de la larva: Psychotria, Faramea

(Rubiaceae) (Maes 1998)

damocrita (Druce, 1894) (Lámina IV)

Departamentos: Cortés, Francisco Morazán, La Paz, Lempira, Ocotepeque (Nuevo registro para Honduras)

Meses de captura: Abril, Julio, Agosto, Septiembre

Altura: $1600-2170 \mathrm{msnm}$

eumedon (Boisduval, 1875)

Departamentos: Francisco Morazán (Nuevo registro

para Honduras)

Meses de captura: Julio

Altura: $1800 \mathrm{msnm}$

germen germen (Schaus, 1890) (Lámina IV)

Departamentos: Cortés, Olancho, Yoro

Meses de captura: Junio, Diciembre

Altura: 1450-1600 msnm
Distribución: México, Guatemala, Nicaragua, Costa Rica, Panamá, Colombia, Ecuador, Bolivia (Maes 1998)

hannemanni Closs, 1917

Distribución: México a Bolivia (Monzón Sierra y Haxaire 2006)

Nota: especie citada por Oehlke (2007)

juanita (Rothschild \& Jordan, 1903)

Departamentos: Colón, Atlántida, Olancho

Meses de captura: Abril, Julio, Octubre

Altura: 1420 msnm

libya (Druce, 1878) (Lámina V)

Departamentos: Atlántida, Francisco Morazán,

Ocotepeque, Olancho, Yoro

Meses de captura: Enero, Mayo, Junio, Agosto

Altura: 35-1200 msnm

Distribución: Sur de Estados Unidos a Bolivia

(Monzón Sierra y Haxaire 2006)

loelia (Druce, 1878)

Departamentos: Atlántida, Cortés (Nuevo registro

para Honduras)

Meses de captura: Febrero, Abril, Junio

Altura: $100 \mathrm{msnm}$

neoptolemus (Stoll, 1780) (Lámina V)

Departamentos: Atlántida, Colón, Gracias a Dios, Olancho, Yoro

Meses de captura: Enero, Marzo, Mayo, Agosto, Septiembre

Altura: 2-1450 msnm

Distribución: México a Brasil central (Monzón Sierra y Haxaire 2006)

pistacina Boisduval, 1875

Departamentos: Atlántida (Nuevo registro para

Honduras)

Meses de captura: Mayo

Altura: $185 \mathrm{msnm}$

Distribución: México a Paraguay (Monzón Sierra y Haxaire 2006)

pluto (F., 1777) (Lámina V)

Departamentos: Atlántida, Cortés, Francisco Morazán, Ocotepeque, Olancho, Santa Bárbara, Yoro Meses de captura: Febrero, Mayo, Julio, Agosto, Septiembre, Diciembre

Altura: 2-1150 msnm

Distribución: Estados Unidos, Las Antillas, México, Nicaragua, Costa Rica, Argentina (Maes 1998)

Hospederos de la larva: Erythroxylon (Erythroxylaceae), Hamelia, Chiococca (Rubiaceae) (Maes 1998) 
porcus continentalis Rothschild \& Jordan, 1903 (Lámina V)

Departamentos: Atlántida, Cortés, Francisco Morazán, Ocotepeque, Olancho, Santa Bárbara, Yoro Meses de captura: Marzo, Abril, Mayo, Julio, Agosto, Septiembre

Altura: 35-1600 msnm

Hospederos de la larva: Palicourea grandifolia (Willd. ex Roem. \& Schult.) Standl. (Rubiaceae) (Hodges 1971)

pyrrhus (Rothschild \& Jordan, 1906)

Departamentos: Atlántida, Olancho (Nuevo registro para Honduras)

Meses de captura: Febrero, Marzo, Mayo, Junio, Julio, Diciembre

Altura: 100-1420 msnm

tersa (L., 1771) (Lámina V)

Departamentos: Atlántida, Cortés, Francisco

Morazán, Ocotepeque

Meses de captura: Enero, Mayo, Agosto

Altura: 2-850 msnm

Hospederos de la larva: Spermacoce glabrila Michx., Pentas, Manettia (Rubiaceae) (Hodges 1971, Maes 1998)

thyelia (L., 1758) (Lámina V)

Departamentos: Atlántida, Santa Barbara, Yoro

(Nuevo registro para Honduras)

Meses de captura: Abril, Septiembre

Altura: 773-1215 msnm

Distribución: México y Centroamérica (Monzón

Sierra y Haxaire 2006)

titana (Druce, 1878) (Lámina V)

Departamentos: Atlántida, Francisco Morazán, Gracias a Dios, Olancho, Santa Bárbara, Yoro (Nuevo registro para Honduras)

Meses de captura: Junio, Abril, Septiembre, Diciembre

Altura: 60-1450 msnm

turbata (Edwards, 1887)

Distribución: México a Venezuela (Monzón Sierra y

Haxaire 2006)

Nota: especie citada por Oehlke (2007)

tyndarus tyndarus (Boisduval, 1875) (Lámina V)

Departamentos: Atlántida, Cortés, Olancho

Meses de captura: Mayo, Agosto

Altura: 35-900 msnm

undata (Rothschild \& Jordan, 1903) (Lámina V)

Departamentos: Gracias a Dios (Nuevo registro

para Honduras)
Meses de captura: Abril

Altura: 60-245 msnm

zurcheri (Druce, 1894) (Lámina V)

Departamentos: Atlántida, Gracias a Dios (Nuevo registro para Honduras)

Meses de captura: Abril, Mayo, Junio, Agosto

Altura: 60-550 msnm

\section{Tribu Philampelini Burmeister, 1878}

Eumorpha Hübner, 1806

achemolus (Cramer, 1779) (Lámina VI)

Departamentos: Atlántida, Colón, Intibucá, Olancho, Santa Bárbara, Yoro

Meses de captura: Enero, Marzo, Abril, Mayo, Junio, Agosto, Septiembre

Altura: 35-1750 msnm

Distribución: México, Nicaragua, Trinidad, Guiana

Francesa, Ecuador, Bolivia, Brasil, Uruguay,

Argentina (Maes 1998)

achemon Drury, 1773

Distribución: Estado Unidos y México (D’ Abrera 1986)

Nota: especie citada por Oehlke (2007)

capronneri (Boisduval, 1875)

Distribución: Venezuela, Amazonas a Guyana (D'

Abrera 1986)

Nota: especie citada por Oehlke (2007)

fasciatus (Sulzer, 1776) (Lámina VI)

Departamentos: Francisco Morazán

Meses de captura: Mayo

Altura: $850 \mathrm{msnm}$

Distribución: Canadá, Estados Unidos, México, Nicaragua, Costa Rica, Uruguay, Argentina (Maes 1998)

Hospederos de la larva: Jussiae, Oenothera, Ludwigia, Fuchsia, Ipilopium (Onagraceae), Vitis, Ampelopsis (Vitaceae) (Maes 1998)

Nota: En los últimos estadíos larvales cambian a diferentes colores, variando desde verde amarillento hasta a verde, de amarillo y rojo, amarillo y verde, y verde y rojo. El cuerno es remplazado por un lóbulo corto. La cabeza posee dos pares de bandas laterales (Moss 1912).

labruscae (L., 1758) (Lámina VI)

Departamentos: Atlántida, Francisco Morazán

Meses de captura: Abril, Mayo, Junio

Altura: 2-850 msnm

Distribución: Estados Unidos, Las Antillas, México, 
Nicaragua, Costa Rica, Uruguay, Argentina, Chile (Maes 1998)

Hospederos de la larva: Eupatorium (Asteraceae), Vitis, Ampelopsis, Cissus (Vitaceae) (Maes 1998), Eupatorium odoratum L. (Asteraceae) (Hodges 1971)

Nota: La larva posee en el tercer segmento torácico un par de manchas con apariencia de ojos. Como mecanismo de defensa, retrae la cabeza y el primer y segundo segmentos torácicos en el tercer segmento, dando como resultado que los ojos parezcan los de una serpiente (Hodges 1971).

obliquus obliquus (Rothschild \& Jordan, 1903)

Distribución: México a Argentina (Monzón Sierra y Haxaire 2006)

Nota: especie citada por Oehlke (2007)

phorbas (Cramer, 1775) (Lámina VI)

Departamentos: Atlántida (Nuevo registro para Honduras)

Meses de captura: Mayo

Altura: 35-185 msnm

Distribución: México, Nicaragua, Costa Rica, Venezuela, Guyana Francesa, Ecuador, Bolivia, Brasil (Maes 1998)

satellitia licaon (Cramer, 1775) (Lámina VI)

Departamentos: Francisco Morazán, Olancho, Yoro

Meses de captura: Mayo, Septiembre

Altura: 420-1215 msnm

Distribución: Estados Unidos, México, Belice, Nicaragua, Costa Rica, Panamá, Colombia, Ecuador, Brasil, Bolivia (Maes 1998)

Hospederos de la larva: Vitis, Ampelopsis, Cissus (Vitaceae) (Maes 1998)

triangulum (Rothschild \& Jordan, 1903) (Lámina VI)

Departamentos: Atlántida, Cortés

Meses de captura: Abril, Junio, Noviembre

Altura: $175 \mathrm{msnm}$

Distribución: México, Nicaragua, Bolivia (Maes 1998)

typhon (Klug, 1836)

Departamentos: Atlántida

Distribución: sur de Arizona (Estados Unidos) a

Honduras (Hodges 1971)

Meses de captura: Abril

vitis vitis (L., 1748) (Lámina VI)

Departamentos: Atlántida, Cortés, Francisco

Morazán, Olancho

Meses de captura: Mayo, Julio

Altura: 185-1600 msnm
Distribución: a través de la región neotropical (Hodges 1971)

Hospederos de la larva: Vitis, Cissus (Vitaceae)

(Maes 1998)

\section{Subfamilia Smerinthinae Grote \& Robinson, 1865 Tribu Ambulycini Butler, 1876}

Adhemarius Oiticica Filho, 1939

dariensis (Rothschild \& Jordan, 1903) (Lámina VII)

Departamentos: Cortés, Francisco Morazán, Olancho

(Nuevo registro para Honduras)

Meses de captura: Junio, Julio, Agosto

Altura: $1450 \mathrm{msnm}$

donysa (Druce, 1889) (Lámina VII)

Departamentos: Atlántida, Comayagua, Cortés,

Francisco Morazán, Intibucá, La Paz, Ocotepeque,

Olancho, Yoro (Nuevo registro para Honduras)

Meses de captura: Enero, Marzo, Abril, Junio, Julio,

Agosto, Septiembre, Diciembre

Altura: 35-2295 msnm

gannascus gannascus (Stoll, 1790) (Lámina VII)

Departamentos: Atlántida, Cortés, Francisco

Morazán, La Paz, Ocotepeque, Olancho, Santa

Bárbara, Yoro

Meses de captura: Enero, Marzo, Abril, Junio,

Agosto, Diciembre

Altura: 35-2170 msnm

Hospederos de la larva: Ocotea (Lauraceae) (Maes 1998)

ypsilon (Rothschild \& Jordan, 1903) (Lámina VII)

Departamentos: Atlántida, Cortés, Olancho, Santa

Bárbara, Yoro (Nuevo registro para Honduras)

Meses de captura: Marzo, Abril, Junio, Julio,

Septiembre

Altura: 175-1450 msnm

Protambulyx Rothschild \& Jordan, 1903

strigilis (L.1771) (Lámina VII)

Departamentos: Atlántida, Cortés, Francisco Morazán, Olancho, Santa Bárbara, Yoro

Meses de captura: Enero, Abril, Mayo, Julio, Agosto

Altura: 5-1600 msnm

Distribución: Centro y Sur América (Hodges 1971)

Hospederos de la larva: Astronium, Spondias, Anacardium (Anacardiaceae) (Maes 1998), Erythroxylom, Comocladia (Erythroxylaceae) (Hodges 1971)

Nota: La larva es verde amarillenta con bandas amarillas oblicuas a los lados (Carter 1992). 


\section{Subfamilia Sphinginae Latreille, 1802 Tribu Acherontiini Boisduval, 1875}

Agrius Hübner, 1819

cingulata (F., 1775) (Lámina VII)

Departamentos: Cortés, Francisco Morazán, Ocotepeque, Olancho, Valle

Meses de captura: Mayo, Agosto, Octubre

Altura: 850-1800 msnm

Distribución: Sur y Centro América, sur de los Estados Unidos, Hawaii (Carter 1992)

Hospederos de la larva: Ipomoea, Merremia (Convolvulaceae) (Maes 1998)

\section{Tribu Sphingini Latreille, 1802}

Amphimoea Rothschild \& Jordan, 1903

walkeri (Boisduval, 1875) (Lámina VIII)

Departamentos: Atlántida, Yoro

Meses de captura: Septiembre

Altura: 5-640 msnm

Hospederos de la larva: Jatropha (Euphorbiaceae)

(Maes 1998)

\section{Cocytius Hübner, 1816}

antaeus (Drury, 1773) (Lámina VIII)

Departamentos: Atlántida, Cortés, Francisco

Morazán, Yoro

Meses de captura: Mayo, Julio, Noviembre

Altura: 850-1600 msnm

Distribución: Estados Unidos, Cuba, Puerto Rico, Jamaica, Bahamas, Martinica, México, Guatemala, Nicaragua, Costa Rica, Surinam, Brasil, Uruguay, Argentina (Maes 1998)

Hospederos de la larva: Annona (Annonaceae) (Maes 1998)

Nota: Es la mariposa más grande de la familia Sphingidae (Carter 1992).

duponchel (Poey, 1832)

Departamentos: Atlántida

Meses de captura: Marzo, Abril

Altura: $100 \mathrm{msnm}$

Distribución: sur de los Estados Unidos a Argentina (Monzón Sierra y Haxaire 2006)

lucifer Rothschild \& Jordan, 1903 (Lámina VIII)

Departamentos: Atlántida, Cortés, Francisco

Morazán

Meses de captura: Mayo, Agosto

Altura: 850-1600 msnm
Hospederos de la larva: especies Annonaceae (Maes 1998)

Manduca Hübner, 1807

dilucida (H. Edwards, 1887)

Distribución: norte de México a Venezuela (Monzón

Sierra y Haxaire 2006)

Nota: especie citada por Oehlke (2007)

incisa (Walker, 1856)

Distribución: Brasil y Bolivia (Oehlke, 2007)

Nota: especie citada por Oehlke (2007)

florestan (Stoll, 1782) (Lámina VIII)

Departamentos: Atlántida, Francisco Morazán, Yoro.

Meses de captura: Mayo, Septiembre

Altura: 35-1450 msnm

Distribución: Estados Unidos, México, Nicaragua, Trinidad, Brasil, Paraguay, Argentina (Maes 1998)

Hospederos de la larva: Citharexylum (Verbenaceae), Tecoma (Bignoniaceae) (Hodges 1971)

hannibal (Cramer, 1779) (Lámina VIII)

Departamentos: Atlántida

Meses de captura: Enero

Altura: $2 \mathrm{msnm}$

lanuginosa (Edwards, 1887)

Departamentos: Atlántida

Meses de captura: Abril

Distribución: norte de México hasta Venezuela

(Monzón Sierra y Haxaire 2006)

lefebrerourii (Guèrin-Mèneville, 1844) (Lámina VIII)

Departamentos: Atlántida

Meses de captura: Junio

Altura: $300 \mathrm{msnm}$

muscosa (Rothschild \& Jordan, 1903) (Lámina VIII)

Departamentos: Atlántida, Yoro

Meses de captura: Septiembre

Altura: 1000-1215 msnm

Distribución: Costa Rica, Estados Unidos (Hodges 1971)

occulta (Rothschild \& Jordan, 1903)

Departamentos: Atlántida

Meses de captura: Marzo Abril.

Altura: 50-100 msnm

Hospederos de la larva: especies de Solanaceae (Hodges 1971)

ochus (Klug, 1836) (Lámina IX)

Departamentos: Atlántida, Francisco Morazán, Olancho, Yoro

Meses de captura: Julio, Septiembre

Altura: 640-1000 msnm 
Distribución: Nicaragua? Colombia, Venezuela?, Ecuador (Maes 1998)

pellenia (Herrich-Schäffer, 1854) (Lámina IX)

Departamentos: Olancho, Yoro (Nuevo registro para Honduras)

Meses de captura: Mayo, Septiembre

Altura: 175-1450 msnm

rustica rustica (F., 1775) (Lámina IX)

Departamentos: Atlántida, Francisco Morazán, Yoro.

Meses de captura: Abril, Mayo, Septiembre

Altura: 640-850 msnm

Hospederos de la larva: Amphilophium, Bignonia,

Pithecoctubreenium (Bignoniaceae), Cordia

(Boraginaceae), Merremia, (Convolvulaceae), Hyptis

(Labiaceae), Lantana, Stachytarpheta, Callicarpa

(Verbenaceae) (Maes 1998)

sexta (L., 1763) (Lámina IX)

Departamentos: Atlántida, Cortés, Francisco

Morazán, Olancho, Yoro

Meses de captura: Enero, Mayo, Septiembre

Altura: 2-1000 msnm

Distribución: Norte y Centro América (Carter 1992)

Hospederos de la larva: Manihot (Euphorbiaceae), Solanum, Nicotiana, Lycopersicon, Capsicum (Solanaceae) (Maes 1998)

Nota: Es un vector de Pseudomonas angulata (Fromme \& Murray) Stevens (angular leaf spot of tobacco) y Pseudomonas tabaci (Wolf \& Foster) Stevens (wild fire of tobacco) en Nicotiana (Maes 1998). Cotesia congregata es un enemigo natural de la larva (Cave 1995).

wellingi Brou, 1984

Distribución: desde Quintana Roo, México hasta

Belice (Oehlke 2007)

Nota: especie citada por Oehlke (2007)

Neococytius Hodges, 1971

cluentius (Cramer, 1775)

Departamentos: Atlántida

Meses de captura: Abril

Sphinx L., 1758

leucophaeta Clemens, 1859 (Lámina IX)

Departamentos: El Paraíso (Nuevo registro para

Honduras)

Meses de captura: Septiembre

merops Boisduval, 1870 (Lámina IX)

Departamentos: Atlántida, Cortés, Francisco

Morazán, Olancho, Yoro
Meses de captura: Enero, Marzo, Mayo, Junio,

Agosto, Septiembre

Altura: 2-1450 msnm

praelongus (Rothschild \& Jordan, 1903) (Lámina IX)

Departamentos: Intibucá

Meses de captura: Agosto

Altura: $1750 \mathrm{msnm}$

\section{Discusión}

Se documentan 107 especies de Sphingidae en Honduras, clasificadas en 28 géneros. Treinta y tres especies son registros nuevos para Honduras. La subfamilia Macroglossinae está representada por 80 especies en 20 géneros; la subfamilia Sphinginae posee 22 especies en seis géneros; la subfamilia Smerinthinae tiene cinco especies en dos géneros. La diversidad de sphíngidos de Honduras es menor a las documentadas en los países vecinos. Maes y Schmit (1999) reportan 119 especies de Nicaragua. En Guatemala, Monzón Sierra y Haxaire (2006) reportan 137 especies en 34 géneros. Es posible que con mayor colecta en las áreas poco muestreadas, como el bosque seco y las áreas protegidas de difícil acceso, el número de especies conocidas de Honduras sea mayor y más complementaria con las faunas de sus países vecinos.

Mayo y Septiembre son los meses en los que se han recolectado el mayor número de especimenes (Figura 3). Posiblemente esto se deba a que Mayo es el mes del año en que normalmente comienzan las lluvias y la vegetación comienza a crecer, proporcionando alimento a las larvas. El elevado número de ejemplares recolectados durante Septiembre puede deberse a que los adultos provenientes de la segunda generación emergen durante este periodo del año. La actividad de vuelo es menor de Octubre a Diciembre.

El Parque Nacional Pico Bonito parece tener la mayor diversidad de especies (Figura 4). Esto puede deberse a la diversidad de la vegetación que el parque posee. Según la clasificación UNESCO (Mejía Ordoñez y House 2002), Pico Bonito posee cuatro ecosistemas vegetales. El Parque Nacional La Muralla tiene la menor cantidad de especies registradas.

Los departamentos de Atlántida, Yoro, Francisco Morazán, y Cortés tienen la mayor diversidad de Sphingidae en Honduras (Figura 5). Sin embargo, 
estos son los más explorados porque los cuatro parques nacionales más recolectados están en estas zonas. El departamento de Francisco Morazán posee también un número alto de ejemplares debido a las colectas realizadas por los estudiantes de la Escuela Agrícola Panamericana en el Valle de Zamorano y el Cerro Uyuca. En el departamento de Atlántida se recolectó en el Parque Nacional Pico Bonito, Reserva de Vida Silvestre Cuero y Salado, y el Jardín Botánico Lancetilla; en el departamento de Yoro las colectas se concentraron en el Parque Nacional Pico Pijol y Parque Nacional Pico Bonito. El mayor número de especies del departamento de Cortés provienen del Parque Nacional Cusuco. Por esta razón es importante hacer notar que las colectas se han realizado en áreas muy específicas y se deben realizar más colectas y revisiones para esta familia en el país, especialmente en los departamentos con menos registros, tales como Comayagua, Intibucá, La Paz, Lempira, Copán, El Paraíso y Valle.

Se encuentra mayor diversidad a las alturas bajas y la diversidad de especies disminuye con el aumento de la altura (Figura 6). De las 88 especies para las cuales tenemos datos de altura, $73 \%$ vive a $<500$ msnm. A las alturas de 1001-1500 msnm, se encuentra al menos $47 \%$. Solamente $3 \%$ existe a $>2000 \mathrm{msnm}$. Cuatro especies (A. donysa, A. gannascus gannascus, $E$. gorgon y $X$. ceratomoides) se distribuyen de 35$>2000$ msnm. Cuatro especies (C. spuria, S.praelongus, $X$. damocrita y $X$. eumedon) se encuentran únicamente arriba de $1500 \mathrm{msnm}$.

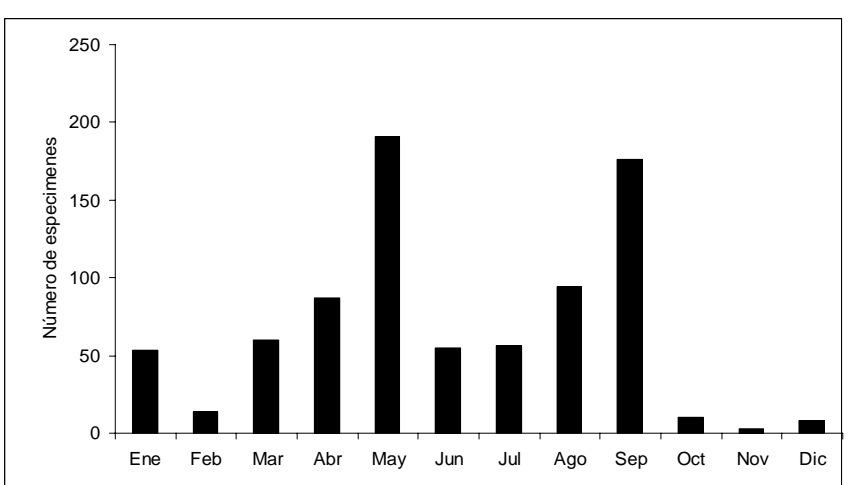

Figura 3. Número de especimenes de Sphingidae capturado por mes en Honduras.

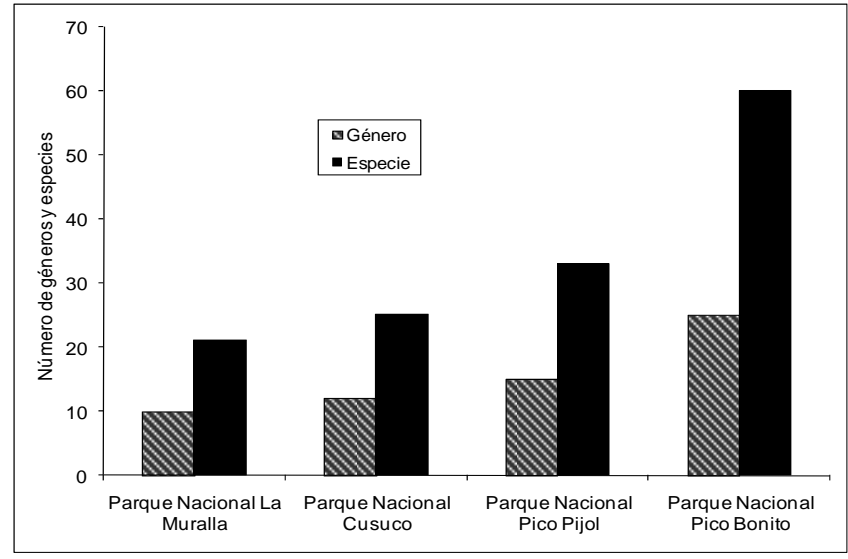

Figura 4. Número de géneros y especies de Sphingidae encontradas en cuatro parques nacionales de Honduras.

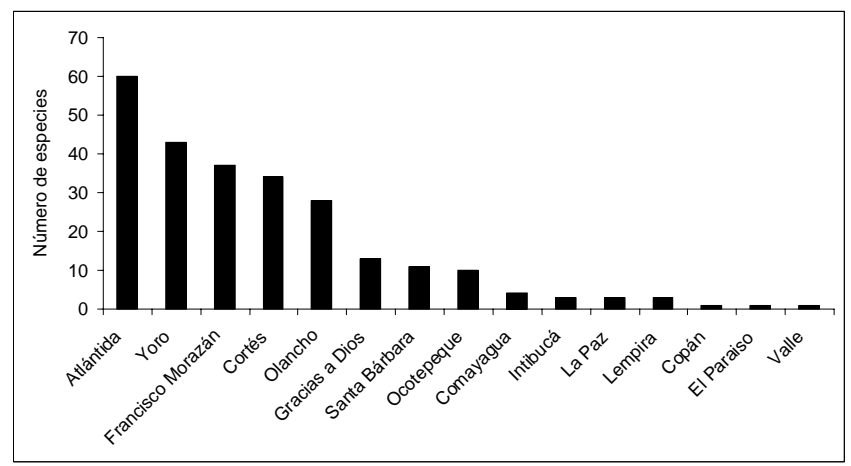

Figura 5. Número de especies de Sphingidae encontradas por departamento de Honduras.

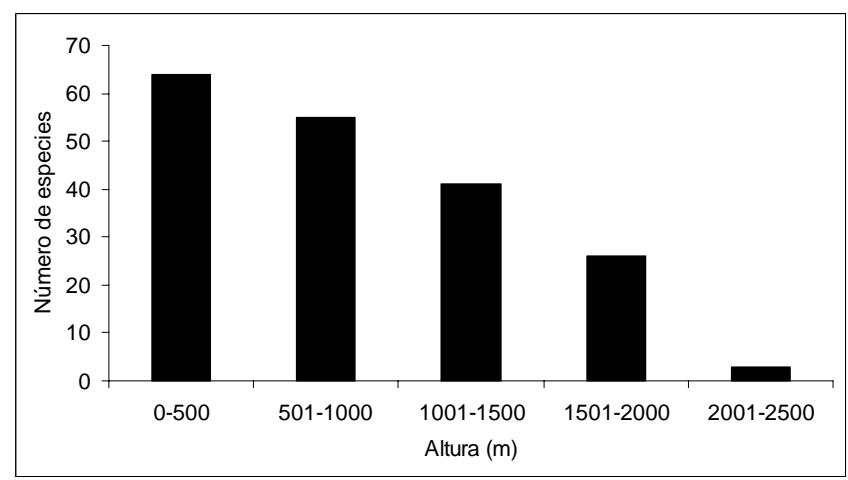

Figura 6. Diversidad de especies de Sphingidae por rango de altura sobre el nivel del mar. 


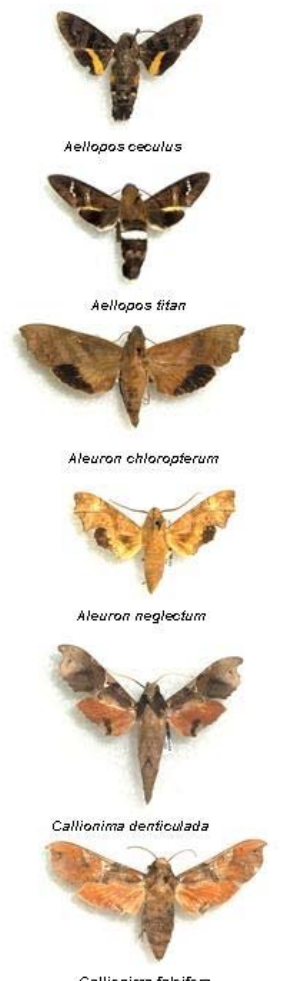

Callionima fakifiera
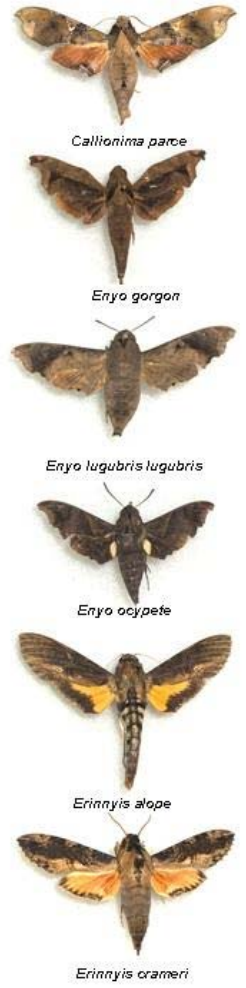

Lámina I. Doce especies de Sphingidae de Honduras.

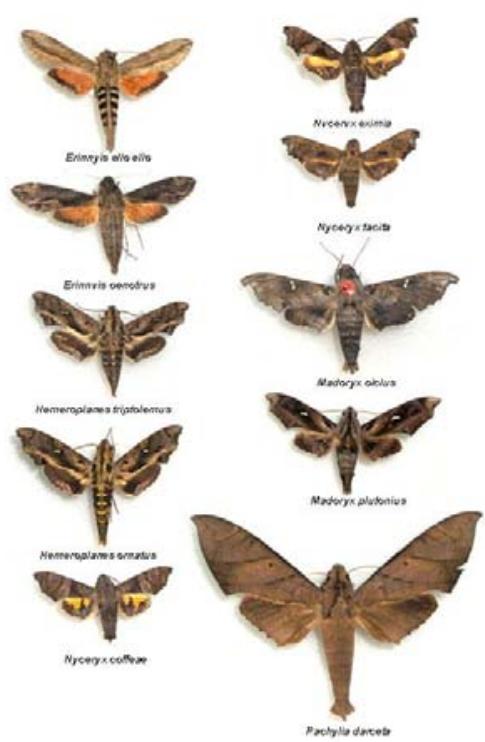

Lámina II. Diez especies de Sphingidae de Honduras.
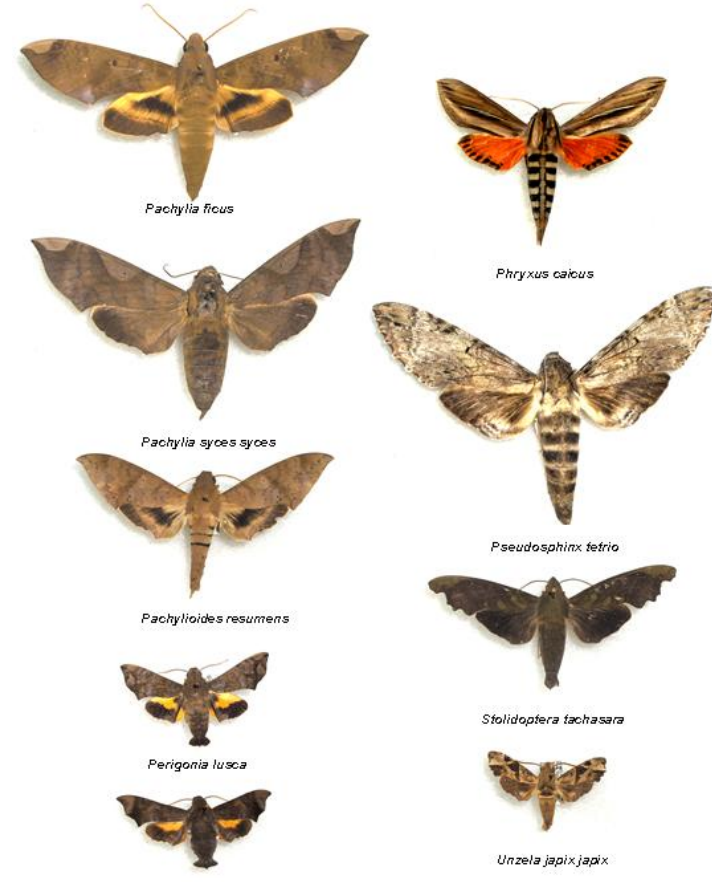

Perigonia stulta

Lámina III. Nueve especies de Sphingidae de Honduras.
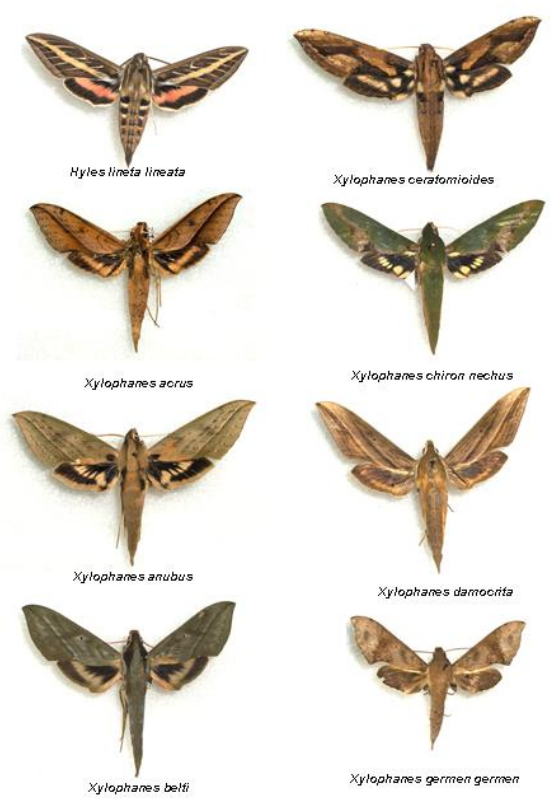

Lámina IV. Ocho especies de Sphingidae de Honduras. 


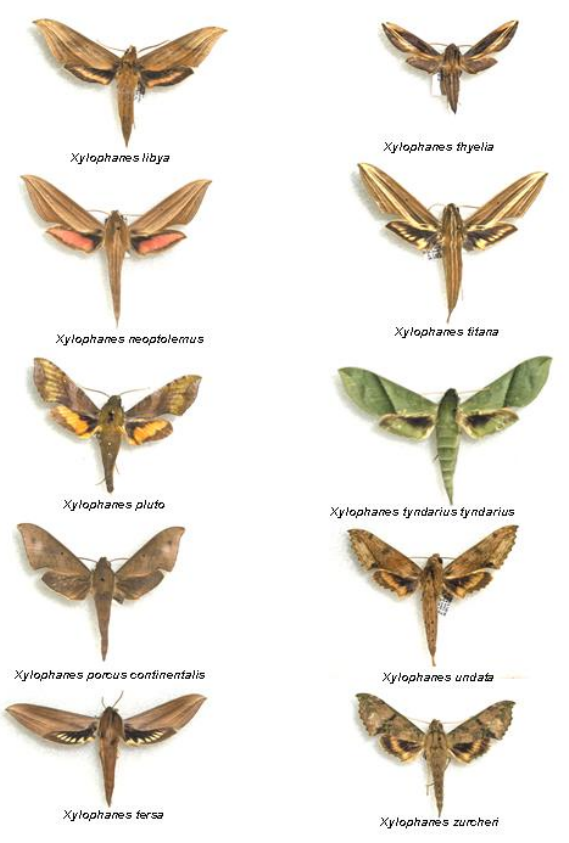

Lámina V. Diez especies de Sphingidae de Honduras.

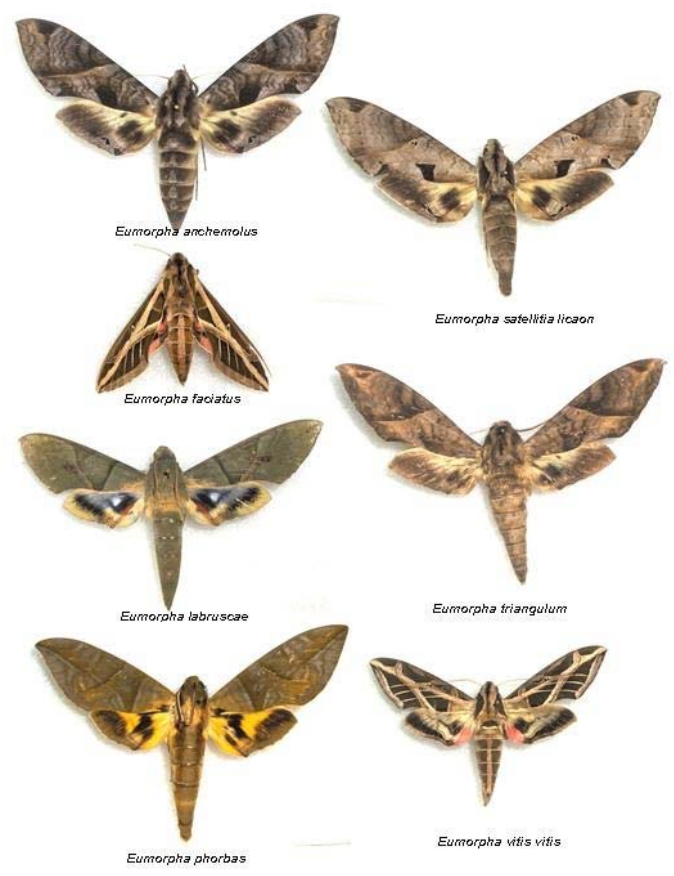

Lámina VI. Siete especies de Sphingidae de Honduras.

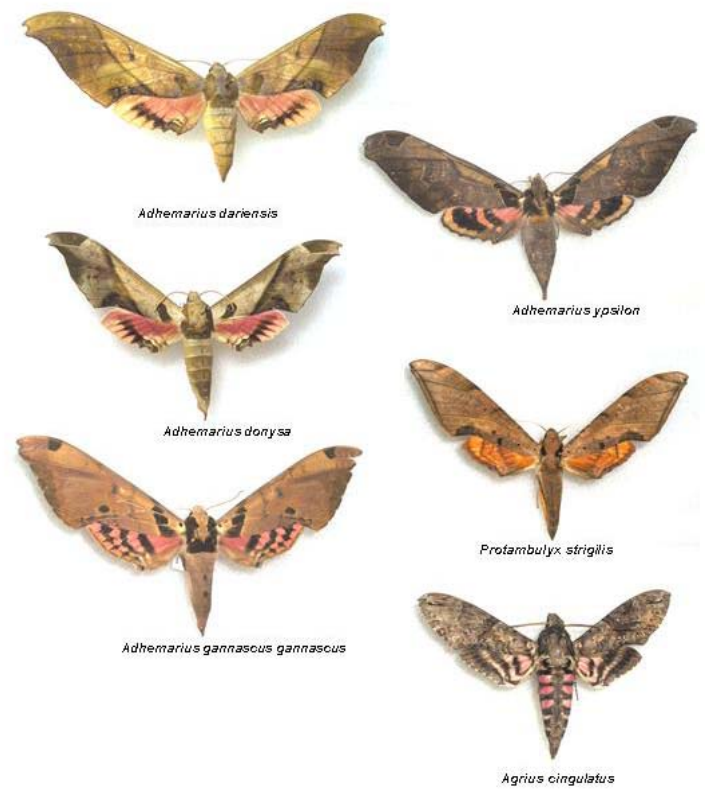

Lámina VII. Seis especies de Sphingidae de Honduras.

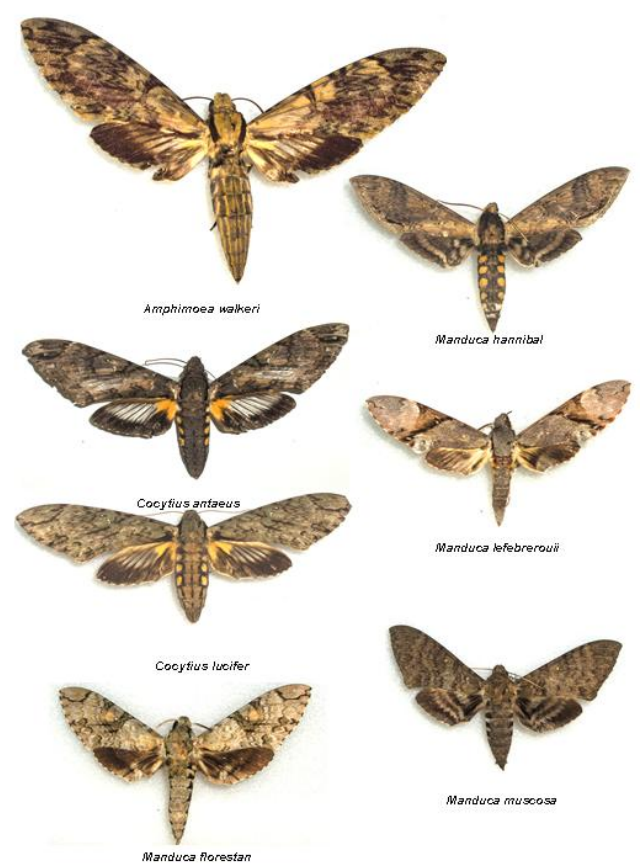

Lámina VIII. Siete especies de Sphingidae de Honduras. 


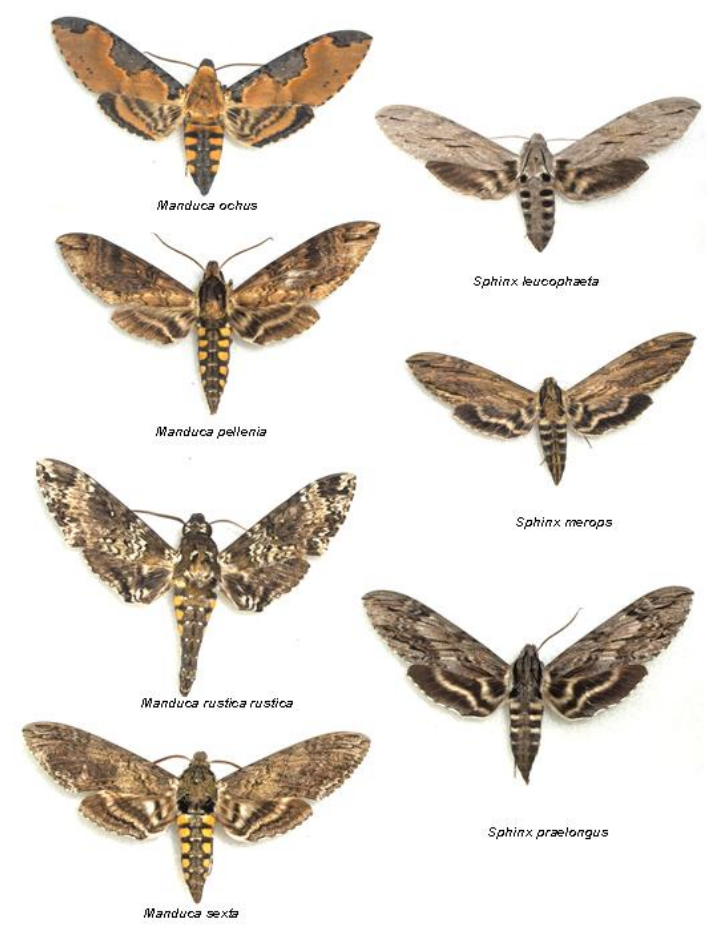

Lámina IX. Siete especies de Sphingidae de Honduras.

\section{Agradecimientos}

Agradecemos a Alfredo Rueda por permitirnos el acceso a la colección de artrópodos de El Zamorano, a Robert Lehman, dueño y curador del Museo de Mariposas en la Ceiba, por permitir el acceso a dicha colección y revisar el manuscrito, a Rodrigo Díaz por ayudarnos a desarrollar el mapa departamental y a Jean-Michel Maes, Edwin Flores y William Oehlke por sus comentarios sobre el manuscrito. Agradecemos a la Fundación Parque Nacional Pico Bonito, Fundación Cuero y Salado y Corporación Hondureña de Forestales por facilitarnos los permisos y el acceso a las áreas protegidas. Este proyecto fue financiado en parte por Fundación VIDA y USAID.

\section{Literatura Citada}

Arnett, R.H. 1985. American Insects: A Handbook of the Insects of America North of Mexico. Van Nostrand Reinhold Company, New York, New York. 850 p.
Carter, D. 1992. Eye Witness Handbooks Butterflies and Moths. First American Edition, DK Publishing Book, New York, New York. 304 p.

Cave, R.D. 1995. Manual para el Reconocimiento de Parasitoides de Plagas Agrícolas en América Central. Zamorano Academic Press, Zamorano, Honduras. 202 p.

D'Abrera, B. 1987. Sphingidae Mundi. The Hawk Moths of the World. E. W. Classey Ltd., United Kingdom. 226 pp.

Hodges, R. W. 1971. The Moths of America North of Mexico. Sphingoidea. En: Dominick, R.B. et al., E.W. Classey Ltd., London, England. fascicle 21: xii + 158 p.

Janzen, D.H. y W. Hallwachs. 2004. Área de Conservación Guanacaste (ACG), Costa Rica http://janzen.sas.upenn.edu/caterpillars/database.lasso. Accesada noviembre 2006.

Kitching, I.J. y J.M. Cadiou. 2000. Hawkmoths of the World, An Annotated and Illustrated Revionary Checklist (Lepidoptera: Sphingidae). Cornell University Press, Ithaca, New York. 226 p.

Lehman R. 1971. Some Sphingidae of Honduras. Journal of the Lepidopterists Society 25: 150-152.

Maes, J.M. 1998. Insectos de Nicaragua, SETAB BOSAWAS, MARZOENA, Managua, Nicaragua, 1,898 p.

Maes, J.-M y P. Schmit. 1999. Fauna Entomológica de Nicaragua, http://www.bionica.org/ento/lepido/sphingidae/sphingidae.htm. Accesada febrero 2007.

Mejía Ordoñez, T.M. y P. House. 2002. Mapa de Ecosistemas Vegetales de Honduras Manual de Consulta. http://wbln0018.worldbank.org/MesoAm/UmbpubHP.ns f/917d9f0f503e647e8525677c007e0ab8/6272461098016 1ea852569a7001e7896/\$FILE/Manual\%20Mapa\%20Ec osistemas.pdf Accesada Agosto 2007.

Monzón Sierra, J. y J. Haxaire. 2006. La familia Sphingidae (Lepidoptera) en Guatemala. p. 423-429. En E.B Cano (ed.) Biodiversidad de Guatemala, Vol. I, Universidad del Valle de Guatemala, Guatemala, Guatemala. 674 p.

Moss, M. 1912. On the Sphingidae of Peru. Transactions of the Zoological Society of London 20: 73-134.

Moss, M. 1920. Sphingidae of Pará, Brazil. Novit Zool. 27(2): 333-424.

Oehlke, B. 2007. Sphingidae of the Americas, http://www.silkmoths.bizland.com/danjansphinx.htm. Accesada Agosto 2007.

Recibido para publicación el 26 de noviembre de 2007. 